p. 227 . $1908 \mathrm{DP}=635$ [1907 ZS] 1908 Sept. 20 Beobachtungszeit $12^{\mathrm{h}} 12^{\mathrm{m}} 41^{\mathrm{s}}$ und 1. f. $\mathrm{p}_{\alpha} 9.282$.

Bd. I84.

p. $32 \mathrm{I}$. 50 Virginia 1909 Dez. 6 Beobachtungszeit $\mathrm{I}^{\mathrm{h}} \mathrm{I}^{\mathrm{m}} 4 \mathrm{I}^{\mathrm{s}}$ $A \alpha+8.33 \alpha 4^{\mathrm{h}} 24^{\mathrm{m}} 55^{\mathrm{s}} .80$ l. f. p. 8.930 und 0.666 .

p. 321. 3I3 Chaldaea rgog Juli $26 A \delta+x^{\prime} 56^{\prime \prime} 5$ und demzufolge $\delta-3^{\circ}$ I $3^{\prime} 40^{\prime \prime} .7$.

p. 323. 478 'Tergeste I 909 März I $9 \Delta \delta+3^{\prime} 24^{\prime \prime} 7$ und demzufolge $\delta$ - I $5^{\circ} 59^{\prime}$ I 2 ." I.

p. 325. 589 Croatia 1909 Dez. $7 \Delta \alpha+\mathrm{I}^{\mathrm{m}} 43^{\mathrm{s}} \cdot 2 \mathrm{I}$ und demzufolge $\alpha 2^{\mathrm{h}} 59^{\mathrm{m}} 22^{\mathrm{s}} .70$ und l. f. p. 8.803 .

Bd. 188.

p. 8I. I I I Ate I 9 Io Jan. 4 Beobachtungszeit $13^{\mathrm{h}} 8^{\mathrm{m}} 7^{\mathrm{s}}$, l. f. p. $9.064 \mathrm{n} 0.674$. Jan. 7 Beobachtungszeit $12^{\mathrm{h}} 14^{\mathrm{m}} 39^{\mathrm{s}}$.

Wien, I 9 I 3 März. p. 85.438 Zeuxo I 9 Io Okt. I 4 Beobachtungszeit I I $^{\mathrm{h}} 2 \mathrm{O}^{\mathrm{m}} 49^{\mathrm{s}}$ I. f. $p_{\alpha} 8.675 \mathrm{n}$.

p. 85 . 550 Senta I 9 Iо März $3 \Delta \delta+I^{\prime}$ I 8.6 und demzufolge $\delta+7^{\circ} 6^{\prime} 22.3$.

p. 97. * ror $16^{\mathrm{h}} \mathrm{I} \mathrm{I}^{\mathrm{m}} 5^{5} .25-17^{\circ} 3^{\prime} 34^{\prime \prime}$. I Wien Anschl. und daher

p. 89. $699[\mathrm{r} 9 \mathrm{r} \circ \mathrm{KD}]$ I 9 xoJuni I $\mathrm{I}^{\mathrm{h}} \mathrm{I}_{\mathrm{I}}^{\mathrm{m}} \mathrm{I} 4^{\mathrm{s}} \mathrm{I} 6-\mathrm{I} 7^{\circ} 2^{\prime} 53^{\prime \prime} 7$.

p. 93. 705 [1910 KV] I910 Jan. $22 \lambda \delta-0^{\prime} 5^{\prime \prime} 2$ und demzufolge $\delta+25^{\circ} 45^{\prime} 49^{\prime \prime} 5$.

p. 98 . * I $332^{2} 3^{\mathrm{h}} 15^{\mathrm{m}} 12^{\mathrm{s}} .75-10^{\circ} 40^{\prime} 3^{\prime \prime} 2$ 1 I $\mathrm{m}$ Wiener Anschluß an $23^{\mathrm{h}} \mathrm{II}^{\mathrm{m}}{ }_{46}{ }^{\mathrm{s}} 35-\mathrm{I}^{\circ}{ }_{42^{\prime}} \mathrm{II}^{\prime \prime} 8 \mathrm{Cbr}$ M. 8 I $5 \mathrm{I}$ und demzufolge

p. 92. I 9 I $\circ \mathrm{KS}=474$ Prudentia I 9 Io Okt. I $423^{\mathrm{h}} 14^{\mathrm{m}} 45^{\mathrm{s}} \cdot 53$ $-10^{\circ} 35^{\prime} 55^{\prime \prime} \mathrm{I}$.

F. Palisa.

\title{
Die Breite des Zentrums der Universitäts-Sternwarte Warschau.
}

Die Breite des Zentrums der hiesigen Sternwarte wird von den astronomischen Jahrbüchern folgendermaßen angegeben :
B. A. J.
C. d. T.
$+52^{\circ} 13^{\prime} 5^{\prime \prime} 7$
+52 I 35
Nautical Almanac +52 I 34.7
American Ephemeris +52 I 3 4.7.

Der Mangel an Ubereinstimmung in diesen Daten veranlaßt uns folgendes mitzuteilen:

Die Breite der hiesigen Sternwarte war bestimmt:

a) Im Jahre 1875 . Zentrum der Sternwarte $+5^{\circ}{ }^{\circ} 3^{\prime} 4^{\prime \prime} 6$. (Zinger, Bestimmung der Längen-Differenz zwischen Warschau und Pulkowa im Jahre I875. Memoiren der militärtopographischen Abteilung des Generalstabs. St. Petersburg. Teil 37, p. 6).

b) Von 1890.56 bis 18.93 .48 Breite des östlichen Gebäudes $+52^{\circ}$ I 3. 5.44, von 1892.54 , bis 1806,47 . Rreiue

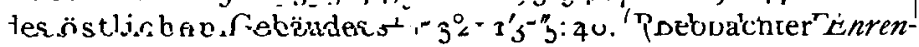
feucht. Th. Albrecht, Bericht über den gegenwärtigen Stand der Erforschung der Breitenvariation. Lausanne 1896. p. 9-I I).

Warschau, Universitäts-Sternwarte, I 9 I 3 März 4.

Reduktion auf das Zentrum der Sternwarte -0.77. Breite des Zentrums der Sternwarte $\left\{\begin{array}{l}+52^{\circ} \text { I } 3^{\prime} 4^{\prime \prime} 67 \\ +52 \text { I } 34.63 .\end{array}\right.$

c) Im Jahre 1897.46 Breite des nördlichen Gebäudes $+52^{\circ}$ I $3^{\prime} 5^{\prime \prime} 43$, im Jahre I 897.55 Breite des nördlichen Gebäudes $+52^{\circ} 13^{\prime} 5^{\prime \prime} 45$, im Jahre 1897.6 I Breite des nördlichen Gebäudes $+52^{\circ}$ I $3^{\prime} 5^{\prime \prime} 48$. (Beobachter Ehrenfeucht. Th. Albrecht, Bericht usw. im Dez. I897.p. 20). Reduktion auf das Zentrum der Sternwarte - 0.90.

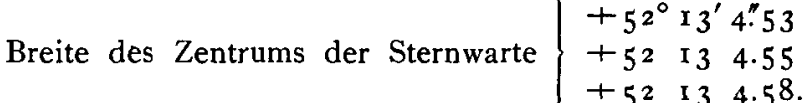

d) Von 1897.46 bis 1898.78 Breite des nördlichen Gebäudes $+5^{\circ}{ }^{\circ} 3^{\prime} 5^{\prime \prime} 54$. (Beobachter Ehrenfeucht. Th. Albrecht, Bericht usw. am Schlusse des Jahres 1898. p. I2). Breite des Zentrums der Sternwarte $+5^{2^{\circ}}$ 1 $3^{\prime} 4^{\prime \prime} 64$.

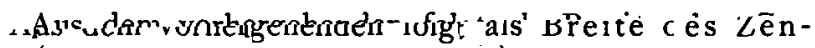
trums'(Merïdian-Kreis Ertel \& Sohn) der UniversitätsSternwarte Warschau

$$
+52^{\circ} 13^{\prime} 4^{\prime \prime} 6 .
$$

\section{S. Tscherny.}

Neuer Veränderlicher 17.1913 Draconis. In M. N. 73.440 teilen $C$. Martin und H. C. Plummer mit, daß der Stern Gr ph $+70^{\circ} 5230$, I $2^{\mathrm{h}} \mathrm{I} 3^{\mathrm{m}} \mathrm{I} 0^{5} 53+70^{\circ} 9^{\prime} 35^{\prime \prime} 3$ ( 1900.0$)$, der als Vergleichstern für SW Draconis gedient hatte, selbst veränderlich ist zwischen $\mathrm{I} 0^{\mathrm{m}} 8 \mathrm{I}$ und $\mathrm{I} \mathrm{m}$. $\mathrm{I} 9$.

Notiz. La Conférence des Ephémérides (Paris I9 I I) ayant décidé que les positions apparentes de plusieurs centaines d'étoiles seraient calculées et publiées par l'Observatoire de Turin, il nous serait très utile de savoir quel nombre d'exemplaires de l'Annuario Astronomico pel I9 5 nous devons faire tirer. Ceux qui le désirent sont priés de s'adresser dès maintenant au Regio Osservatorio di Pino Torinese (Italie). F. Boccardi.

Anzeige. Durch mehrfache Anfragen veranlaßt habe ich mich entschlossen, von jetzt ab auch auf das den Astronom. Nachr. monatlich beigelegte Literarische Beiblatt selbständige Abonnements anzunehmen. Der Abonnementspreis beträgt $6 \mathcal{M}$. für den Jahrgang bei portofreier Zusendung. Der Jahrgang beginnt mit dem I. Juli, die erste Nummer des Jahrganges erscheint daher im August. Von dem demnächst zum Abschluß kommenden Jahrgange ist noch eine Anzahl vollständiger Exemplare vorhanden. Bestellungen sind an die Expedition, Kiel, Moltkestraße 80, oder durch den Buchhandel an Herrn Theod. Thomas, Komm.-Gesch., Leipzig, Talstraße I 3, zu richten.

Kiel, r9 3 Juli 9.

Hermann Kobold.

Inhalt zu Nr. 4666. F. Palisa. Beobachtungen von kleinen Planeten und Kometen an der Wiener Universitätssternwarte im Jahre I9I2. I77. - S. Tscherny. Die Breite des Zentrums der Universitäts-Sternwarte Warschau. 199. - Neuer Veränderlicher I7.ı9ı3 Draconis. 199. - Kleine Mitteilungen. 199. 